\title{
ASSESSING OPHTHALMIC SERVICES
}

\author{
PARUL DESAI \\ London
}

The need for reliable and representative information regarding the quality of ophthalmic services provided in the United Kingdom at either local or national level, has only recently acquired a certain urgency as a result of audit, accountability, the demands of the internal market and the requirement for health authorities to purchase services to meet the needs of the population. ${ }^{1-4}$

An assessment of any service should ideally consider the components that contribute to that service: current clinical practice, the process by which the service is provided and the outcomes of the care that has been provided (for those presenting to the health care services). Population-based estimates of prevalence or incidence of disease, when available, can indicate the need for services in a given community. Routine sources of data within the National Health Service from hospital (e.g. Korner minimum sets) and community information systems, essentially provide process information and limited outcomes information for health services management. Variations in accuracy, completeness and the type of information collected have so far limited the use of these data for assessment of services.

Epidemiology is the basic science of public health medicine. Epidemiological study methods are classically designed to provide an unbiased estimate of the distributions and determinants of disease. The application of these methods for assessment of medical/surgical services allows for the collection of representative data from which inferences may be made to inform public health policy on the provision and planning of services at either the local (district or region) or national level.

For public health purposes, surveys are particularly useful means of obtaining information for needs assessment, and for planning and provision of health care services. A cross-sectional survey is essentially a descriptive epidemiological study. It provides information on the frequency and characteristics of a

Correspondence to: Parul Desai, MSc, FRCS, FRCOphth, London School of Hygiene \& Tropical Medicine (University of London), Keppel Street, London WC1E 7HT, UK. disease by furnishing a 'snapshot' of the health experience of a defined population at a specified time. These data are useful in public health in assessing the health status and health care needs of a population. Applying this design to service assessment, whether locally or nationally, provides information on the frequency of an intervention such as cataract extraction and the characteristics of the surgical process, producing a 'snapshot' of the health care system currently available for patients undergoing cataract surgery. The National Cataract Surgery Survey is an example of a national evaluation of services for age-related cataract using a crosssectional survey design. ${ }^{5}$

By collecting data on the experience of a representative sample of patients undergoing cataract surgery in the United Kingdom in 1990, the National Cataract Surgery Survey described current surgical practice within the National Health Service and related process (provision of medical/surgical care) to the short-term clinical outcomes of cataract surgery. ${ }^{5-7}$

As expected, it not only showed that cataract extraction is a common procedure performed on elderly patients, but was also able to quantify this observation by showing that operation rates were 5 times higher in patients of 75 years of age and over compared with those between 50 and 74 years. Many of these patients have considerable levels of visual impairment, with about 1 in 5 patients having surgery on an eye that is blind from cataract. Good clinical outcome, defined as post-operative visual acuity of $6 / 12$ or better at 3 months post-operatively, was achieved in about $80 \%$ of all patients irrespective of the pre-operative visual acuity. Surgically related complications occurred in about $20 \%$ of patients at clinically significant periods post-operatively.,

Neither the region in which the surgery was carried out, the type of hospital, grade of surgeon performing the operation, the type of admission (day case or in-patient), nor the type of anaesthetic (local or general) were observed to influence short-term clinical outcomes. ${ }^{6}$ In public health terms these 
findings reflect the quality of care provided nationally for patients with age-related cataract.

Since cross-sectional surveys provide data from one point in time they are useful for raising the question of the presence of a possible association rather than for testing a hypothesis for a causal association. For example, operation rates computed directly from survey data demonstrated a twofold difference between the regions with the highest and lowest operation rates. ${ }^{7}$ It is unlikely that there are major differences in the prevalence of cataract across regions, and consequently in the demand for surgery, that could contribute to the variation observed. Although it is not possible to quantify any random variation in activity occurring as a consequence of sampling, other possible factors such as the availability of theatre time, beds, waiting lists initiatives, manpower and other resources may have influenced this observation. While cross-sectional surveys suggest these factors may play a role, meaningful assessment of their influence on operation rates at a particular time cannot be made from the survey data as these factors may change over time.

In summary, the survey has generated data that were previously unavailable for the United Kingdom. It has provided national figures on process and outcome of cataract surgery that could be used for local or regional audit and that could contribute to the development of guidelines for the management of age-related cataract. It represents a national database for cataract surgery which if regularly updated could monitor trends over time in the indications for surgery, surgical techniques, availability and use of resources.

\section{REFERENCES}

1. Secretaries of States for Health, Wales, Northern Ireland and Scotland. Medical audit working paper 6. London: HMSO, 1989.

2. Department of Health. Medical audit in the hospital and community health service. London: DOH, 1991 (HC (91)2).

3. Department of Health. Moving forward: needs assessment, services and contracts. DHA Project March 1991 (EL(91)40).

4. Department of Health. Assessing health care needs: a DHA project discussion paper. NHS Management Executive, May 1991.

5. Courtney P. The National Cataract Surgery Survey. I. Methods and descriptive features. Eye 1992;5:487-92.

6. Desai P. The National Cataract Surgery Survey. II. Clinical outcomes. Eye 1993;7:489-94.

7. Desai $P$. The National Cataract Surgery Survey. III. Process features. Eye 1993;7:667-71. 